\title{
Cooperación e integración cultural como prácticas gerenciales en la gestión de la calidad en la Universidad de La Guajira
}

\section{Cooperation and cultural integration as management practices in the quality management at the Universidad de La Guajira}

DOI: http://dx.doi.org/10.17981/econcuc.39.1.2018.05

Artículo de investigación. Fecha de recepción: 10/11/2017 Fecha de aceptación: 08/03/2018

\author{
Astrid Terán Molina (D) \\ Universidad de La Guajira (Colombia) \\ ateran@uniguajira.edu.co \\ Melissa Paola Consuegra Terán \\ Universidad de La Guajira (Colombia) \\ mconsuegrat@uniguajira.edu.co \\ Olisney De Luque Montaño (D) \\ Universidad de La Guajira (Colombia) \\ odeluquem@uniguajira.edu.co
}

Para citar este artículo:

Terán, A., Consuegra, M. y De Luque, O. (2018). Cooperación e integración cultural como prácticas gerenciales en la gestión de la calidad en la Universidad de La Guajira. Económicas CUC, 39(1). 75-86, 2018 DOI: http://dx.doi.org/10.17981/econcuc.39.1.2018.05

\section{Resumen}

El objetivo consiste en describir las estrategias gerenciales de la Institución Educativa Superior (IES), actor en el desarrollo local, y su inclusión internacional como apalancamiento para alcanzar calidad, proyectarse y ser competitiva. La revisión documental institucional y encuesta dicotómica aplicada a una muestra seleccionada a discreción de los investigadores de 150 personas del estamento universitario (de una población de 16.000 entre estudiantes, docentes y administrativos), mediante instrumento validado por expertos y medida su confiabilidad con el Alpha de Cronbach con un nivel de confianza del $95 \%$ y un margen de error del 8\%, cuyo porcentaje de $\mathrm{P}$ y $\mathrm{Q}$ es de 0,50 , mostró como resultado que cuando se utilizan prácticas gerenciales de internacionalización como la cooperación e integración cultural se da una transformación institucional y se dinamiza el proceso de acreditación y el mejoramiento continuo para la calidad y excelencia.

Palabras clave: Acreditación, calidad, educación, cooperación, internacionalización, integración cultural

\section{Abstract}

The purpose of this paper is to show the management strategies of this HEI as an actor in local development as well as of its international inclusion policy as a leverage strategy to reach quality, to project itself globally and to be competitive. The process was performed by an institutional documentary review and a dichotomous survey applied to a sample selected at the discretion of the researchers of 150 people from the university (from a population of 16,000 among students, teachers and staff). The instrument was validated by experts and its reliability measured with the Alpha of Cronbach with a confidence level of $95 \%$ and an error margin of $8 \%$, whose percentage of $\mathrm{P}$ and $\mathrm{Q}$ is 0.50 . The results showed that when management practices of internationalization, such as cooperation and cultural integration are generated, an institutional transformation occurs and streamlines the accreditation process, thus, encouraging a continuous improvement towards quality and excellence.

Keywords: Accreditation, quality, education, cooperation, internationalization, cultural integration 


\section{Introducción}

La educación terciaria en América Latina enfrenta el reto de responder a las exigencias de la globalización y la sociedad del conocimiento a partir de una situación de rezagos en términos de calidad, pertinencia, cobertura, equidad y financiamiento de la educación superior para generar productividad científica y tecnológica (Bellei, 2013). Por ello, la cooperación e internacionalización son consideradas condición sine qua non ${ }^{1}$ para que las instituciones de educación superior puedan cumplir con el papel social que les corresponde, como es ofrecer la enseñanza, el aprendizaje con calidad, la investigación y el servicio a la sociedad (Komlavi Francisco Seddoh, director de la División de la Educación Superior de la UNESCO, citado en Universidad Militar Nueva Granada, 2011) en razón a su función social y motor del desarrollo.

La internacionalización, aplicada a la educación, hace referencia al conjunto de prácticas y dinámicas que las Instituciones de Educación Superior han desarrollado y efectuado con el objetivo de insertarse en el actual mundo académico, caracterizado por las constantes relaciones de competencia y dinamismo entre los países. Esto trae como resultado mejor calidad en los programas académicos, así como egresados capaces de enfrentar escenarios laborales marcados por la interdependencia de la globalización y profesionales competitivos con amplia visión de mundo (Medina y Guzmán, 2011). Con estos fines, la internacionalización hace exigible el desarrollo de procesos como la evaluación y acreditación con miras a promover una educación con calidad y la competitividad de las Instituciones de Educación Superior ante la diversidad de la oferta. Por ello, "la evaluación y la acreditación han pasado a convertirse en imperativos, ya que garantizan

${ }^{1}$ Condición necesaria, sin la cual no es posible alcanzar los objetivos propuestos en una organización. a la sociedad la calidad y credibilidad de los procesos educativos y sus resultados" (Consejo Nacional de Acreditación-CNA, 2006, p. 11).

Ante tales exigencias, la Universidad de La Guajira observa el manejo de las relaciones públicas, la cooperación, la integración cultural y la asociación mediante el establecimiento de convenios marco con otras Instituciones de Educación Superior en ciudades fronterizas y transfronterizas para el desarrollo de mecanismos de cooperación técnica, científica y académica con el propósito de concretar la calidad una vez se haya alcanzado el fortalecimiento de los procesos de acreditación de los programas de pregrado y la acreditación institucional, basados en el beneficio mutuo entre los actores. De esta manera, estaría a la vanguardia sobre nuevas prácticas de cooperación, intercambios culturales, internacionalización de la investigación, extensión y movilidad académica como herramientas fundamentales que le permitan ser competitiva frente a otras instituciones a nivel mundial.

Con esos razonamientos les surge a las investigadoras el siguiente interrogante: ¿de qué manera la Universidad de La Guajira puede gerenciar la cooperación e internacionalización para apalancar su inclusión en escenarios mundiales? Por lo anterior, se analizaron las prácticas gerenciales y de gestión para la consolidación de la acreditación institucional y lograr una participación importante en los asuntos globales en ejercicio de su autonomía y proyección internacional. En vista de que a la internacionalización se le considera una estrategia clave para responder a las demandas del nuevo contexto mundial (Gacel-Ávila, 2012), este artículo se propone, además de describir los logros, limitaciones, expectativas y retos de la internacionalización dentro del proceso de acreditación institucional en la Universidad de La Guajira, describir las prácti- 
cas gerenciales y los procesos claves de internacionalización como estrategia de apalancamiento para la acreditación y accionar internacional a través de sus redes transnacionales.

Producto de la revisión documental y bibliográfica, se describen los fundamentos teóricos de la internacionalización como estrategia para la acreditación de la educación superior con la que se quiere proveer servicios de calidad. Haciendo uso de los hallazgos de la investigación titulada "Internacionalización una visión estratégica de visibilización y cambio institucional en la Universidad de La Guajira", cuyo objetivo consiste en analizar la internacionalización y los cambios en las prácticas administrativas, se da a conocer la percepción sobre la internacionalización para materializar la acreditación y su proyección en escenarios mundiales. La pertinencia y relevancia de esta investigación radica en identificar posibles barreras y fortalezas que de alguna manera contribuyan a lograr sus propósitos misionales, que tienen como fin último lograr altos estándares de calidad y preparar a sus estudiantes para un mundo globalizado e internacionalizado.

\section{Fundamentación teórica}

El proceso de transformación institucional se ha constituido en un reto para la Universidad de La Guajira con miras a lograr la acreditación y contar con el reconocimiento de alta calidad a nivel nacional e internacional. Los referentes teóricos sobre los que se soporta la internacionalización de la educación superior, son Rosario, Marúm, Vargas, Arroyo y González (2006); De Wit, Jaramillo, Gacel-Avila y Knight (2008); Knight (2001); Ávila y Aguinaga (2013) y Rosario, Didriksson y Marúm (2013), entre otros. Por otra parte, sobre la base que las comunidades académicas pueden establecer nuevos compromisos y seguir políticas de adaptación flexibles a entornos cambiantes, se acude a las perspectivas teóricas en términos de cooperación internacional como prácticas gerenciales en la administración pública.

La internacionalización de la educación superior tiene sus raíces en la Constitución Nacional de 1991 al establecer la educación con calidad para el cumplimiento de sus fines (art. 67); en la garantía de la autonomía universitaria, el fortalecimiento de la investigación científica, y el ofrecimiento de las condiciones especiales para el desarrollo institucional (art. 69). Quiere decir que no se puede gerenciar la internacionalización del servicio educativo si antes no se gestiona la calidad, la cual, aparte de ser compleja es multidimensional. De la Orden (1988), la define como "un sistema de coherencias múltiples (...) por ello se podría aplicar a muy diferentes formas" (p. 152). Aplicada a la educación, para el mismo autor, la calidad educativa es "un continuo cuyos puntos representan combinaciones de funcionalidad, eficacia, eficiencia altamente correlacionados, y su gran máximo, la excelencia, supone un óptimo nivel de coherencia entre todos los componentes fundamentales del sistema" (p. 153).

Para Tobón, Rial, Carretero y García (2006) "la calidad educativa tiene como fines establecer mecanismos para asegurar la pertinencia y pertenencia de los procesos de docencia, investigación y extensión, como funciones centrales de la educación superior" (p. 7). En otras palabras, se requiere disponer de los medios y recursos que posibiliten no solo el desarrollo de las capacidades académicas sino también de la organización.

En ese orden de ideas, para lograr la calidad académica y pertinencia social, la ANUIES $^{2}$ (2006) postula que las instituciones de educación superior deberán considerar estructuras curriculares flexibles,

2 Asociación Nacional de Universidades e Instituciones de Educación Superior. 
movilidad estudiantil, elementos formativos en todos los programas que promuevan la comprensión de los entornos sociales, los derechos humanos, el desarrollo sustentable, la paz, los valores democráticos; la integración de la dimensión cultural e intercultural; programas de emprendimiento; cambios en los métodos educativos; diversificación de los ambientes de aprendizaje; y políticas públicas que se orienten al estímulo de la innovación.

La acreditación en el contexto de la educación superior en Colombia tiene como fin la calidad, la cual hace referencia, según la Ley de Educación Superior, a "los resultados académicos, a los medios y procesos empleados, a la infraestructura institucional, a las dimensiones cualitativas y cuantitativas del servicio prestado y a las condiciones en que se desarrolla cada institución” (Ley 30, 1992). De este modo, se puede entregar a la sociedad centros y programas de educación superior con los más altos estándares de calidad, de donde egresen profesionales con los atributos para incorporarse en las dinámicas globales.

Lo antes visto plantea una cultura de acreditación institucional y no un mero tecnicismo. En efecto, se trata de consolidarse y logar el reconocimiento por su misma pertinencia social; además de ser parte de la política educativa, "representa un espacio para repensar estos procesos [certificación, evaluación y calidad universitaria], reconociendo la identidad, los valores institucionales y contextuales de las propias instituciones educativas" (Rosario, et al., 2006, p. 468).

No obstante, por el fenómeno mismo de la internacionalización, las instituciones de educación superior se han visto obligadas a replantear sus objetivos de acreditación hacia la internacionalización en concordancia con el principio constitucional de educación con calidad, la cual en los últimos tiempos se ha caracterizado por una dimensión internacional que compren- de: "el intercambio de conocimientos, la creación de sistemas interactivos, la movilidad de profesores y estudiantes y los proyectos de investigación internacionales teniendo presente los valores culturales y las circunstancias internacionales" tal como propuso la Unesco en la Declaración Mundial sobre la Educación Superior en el Siglo XXI: Visión y Acción (1998, p. 67, citado por Rosario et al., 2006, p. 17).

Según se ha visto, la incursión en el contexto global, el aprendizaje en el uso de las TIC y el conocimiento de otros idiomas o de una segunda lengua ha incentivado la transformación curricular como un reto en las instituciones de educación superior y el gobierno nacional, asignando singular importancia a la internacionalización. Para Atria (2012), el acuerdo de Bolonia y los requisitos asociados a la movilidad académica han fortalecido el desarrollo de procesos de aseguramiento de la calidad de la educación superior dentro de los cuales se incluye la acreditación institucional y de programas con miras a su internacionalización.

La internacionalización de la educación superior "es el proceso de integrar la dimensión internacional/intercultural en la enseñanza, la investigación y el servicio de la institución" (Knight, 2001, p. 22). De igual modo, plantea que, más que convertirse en un elemento de los programas y políticas de las instituciones de educación superior, han de constituirse en tema y eje principal de una universidad $\mathrm{y}$, por tanto, debe destacarse en los planes de desarrollo institucional. En esa misma dirección se asume la internacionalización de la educación como "la inclusión de una dimensión internacional, intercultural o global en el plan de estudio o en su proceso de enseñanza aprendizaje" (De Wit, Jaramillo, Gacel-Avila y Knight, 2008, p. 2). En razón a ello, el documento marco Reflexiones para la política de internacionalización de educación superior en 
Colombia (Salmi, Martínez, Nupia, LucioArias, Lucio, Langebaek, Téllez, AnzolaPardo, Arango, Cañón, Cruz, Toro, Vélez, De Wit, Prieto, Valderrama, Allain-Munoz y Aponte, 2014) plantea como procesos claves que deben liderarse desde el nivel gerencial una serie de transformaciones relacionadas con la innovación curricular, la movilidad académica y la investigación internacional, los cuales son sujeto de análisis al interior de los resultados.

Bajo esas consideraciones, la universidad pública deberá transformarse para ofertar y operar el comercio de servicios educativos con calidad, bien sea bajo la modalidad de suministro transfronterizo (semipresencial, virtual), consumo de servicios en el extranjero (movilidad académica), la prestación de servicios en un país miembro o la prestación de servicios por profesionales extranjeros. Lo anterior está amparado por la Conferencia Regional de Educación Superior de América Latina y el Caribe (CRES, 1996), donde se debatió sobre las Estrategias para la Transformación de la Educación Superior en América Latina y el Caribe, tocando como ejes importantes la pertinencia, la calidad, el financiamiento y la gestión de la educación superior, y la reformulación de la cooperación internacional.

La relación entre la educación superior y la cooperación internacional está ratificada por el artículo 63 de la Primera Cumbre de Jefes de Estado y de Gobierno de los países de la Unión Europea, América Latina, el Caribe (UEALC), donde se resalta la voluntad de reforzar la cooperación en el ámbito de la educación como reto mayor, insistiendo en la cooperación entre las instituciones de enseñanza superior, respetando su autonomía, especialmente mediante los intercambios universitarios y el desarrollo de la enseñanza a distancia.

En esa dirección se encuentra el papel de los Estados en el marco de la cooperación al desarrollo (Arias y Molina, 2008), fundamento para el establecimiento de los acuerdos y convenios, instrumentos éstos para la gestión académica basados en el principio de cooperación para dinamizar la movilidad de la investigación y el conocimiento. En este orden de ideas, la cooperación y su articulación en el contexto internacional llevan implícito el reconocimiento cultural. En las instituciones de educación superior, la integración cultural y multicultural cobran relevancia al momento de considerar la internacionalización del currículo, por lo que una de sus características comprende la "exigencia de conductas culturalmente inclusivas" (Jones y Brown, 2014, p. 119) y "la valoración de la diversidad y las diferencias culturales y lingüísticas" (Leask, citado por Jones y Brown, 2014, p. 119).

\section{Metodología}

La investigación mixta de tipo descriptiva se aborda desde un diseño no experimental, de campo y documental. Para la recolección de la información se realizó la revisión documental institucional y la aplicación de una encuesta estructurada dicotómica a una muestra de 150 personas de todos los estamentos de la universidad, la cual es una población de 16.000 entre estudiantes, docentes y administrativos ligados a los procesos administrativos de acreditación institucional e internacionalización. Se tuvo un nivel de confianza del $95 \%$ para un valor de $\mathrm{Z}$ de 1,96 y un margen de error del $8 \%$, cuyo porcentaje de $\mathrm{P}$ y Q es de 0,50 (ver formula estadística). El instrumento fue validado por expertos y su confiabilidad medida con el coeficiente de Alpha de Cronbach. En lo que respecta a la interpretación de resultados se utilizó el análisis descriptivo de procesos, documentos y fenómenos encontrados en las prácticas institucionales y el análisis estadístico de frecuencias, medidas de tendencia central y un baremo para la percepción. 


\section{Discusión de los resultados}

Con este artículo se muestra cómo la gerencia de la internacionalización de la educación superior adelantada por la Universidad de La Guajira viene apalancando de manera paralela la acreditación institucional como condición para operar el comercio de servicios educativos con calidad a nivel nacional e internacional mediante estrategias gerenciales, como la cooperación e integración cultural. El camino transitado revela, a partir de la revisión documental de los informes técnicos de autoevaluación (2016) de los programas de licenciatura de la Facultad de Ciencias de la Educación, de evaluación por pares académicos y hallazgos extraídos de la investigación titulada Internacionalización una visión estratégica de visibilización y cambio institucional en la Universidad de La Guajira (2017), resultados importantes sobre la gestión de la internacionalización como uno de los factores que contribuyen a la acreditación de sus programas.

Las Políticas Institucionales, el Plan de Desarrollo y el Proyecto Educativo Institucional conciben la internacionalización como un conjunto de prácticas y dinámicas que la Universidad de La Guajira ha desarrollado con el objeto de insertarse en el mundo académico. La internacionalización de la Universidad de La Guajira se implementa como un proceso que ha permitido generar un cambio en el pensamiento de cada uno de los estamentos que conforman la alma mater, dando paso al reconocimiento de la universidad en el escenario nacional e internacional. En este sentido, la alta dirección en conjunto con la Oficina de Relaciones Internacionales (ORI) institucionalizó la política de internacionalización establecida en el Acuerdo 014 de 2016, la cual ha permitido el desarrollo de mecanismos y estrategias en articulación con las unidades académicas $\mathrm{y}$ administrativas.
Por su parte, la ORI centra su atención en unas áreas específicas de trabajo, tales como la ampliación de alianzas estratégicas, intercambios académicos, proyectos de cooperación internacional e internacionalización curricular. Lo anterior en aras de promover la calidad académica y la acreditación institucional de la Universidad de La Guajira y, así, formar profesionales competentes y capaces de afrontar el ámbito global. La internacionalización en la Universidad de La Guajira se centra en los siguientes propósitos fundamentales: a) internacionalización del currículo, b) convenios y cooperación internacional, c) movilidad académica, d) internacionalización de la investigación y extensión.

De esa manera, se pretende que la internacionalización sea vista como un proceso que debe estar apropiado e incorporado dentro de la planeación de las funciones de docencia, investigación y proyección social. Igualmente, se espera que la generación de múltiples escenarios y condiciones sean propicias para lograr un proceso de internacionalización eficiente y acorde con los lineamientos institucionales de la Universidad de La Guajira.

Al momento de revisar cada uno de los criterios técnicos para la acreditación institucional y cruzándolos de manera transversal con la gestión internacional, se identifican puntos comunes, entre ellos, la búsqueda incesante de mejores condiciones para una educación de calidad. Por la forma como se gerencia la internacionalización, se estima su aporte de manera complementaria a la acreditación institucional. La internacionalización es promovida como un "proceso de transformación institucional e integral siendo incorporada en la misión y funciones sustantivas de docencia, investigación y proyección social, de cada una de sus programas académicos" 3 .

${ }^{3}$ Extraído de la valoración de la inserción del programa en contextos académicos nacionales e internacionales y las relaciones externas de profesores y estudiantes realizado por pares académicos. 
La gestión de la cooperación y las relaciones internacionales como tendencias gerenciales que se adelanta en la universidad contribuyen a impulsar la inserción de sus servicios académicos en el contexto nacional e internacional; las relaciones externas de profesores y estudiantes se adelantan con el apoyo institucional que brinda la Oficina de Relaciones Internacionales, facilitando la movilidad profesoral, estudiantil, directivos de programas y egresados entrantes y salientes en el acceso a eventos nacionales e internacionales en calidad de expositores; y congresos e investigaciones, entre otras actividades, se encuentran como características de la dimensión internacional descritas por Rosario y colaboradores (2006).

Tales aspectos comunes para la acreditación y la internacionalización de la educación en la universidad hacen que las acciones gerenciales orienten sus esfuerzos hacia la inclusión a nivel internacional en países e instituciones de España, México, Perú, Brasil, Venezuela, Argentina, Estados Unidos, Panamá, Cuba, Ecuador, Costa Rica, Chile y Curazao, con los cuales se mantienen acuerdos de cooperación académica, permitiendo explorar nuevos nichos del conocimiento como fines de la movilidad de docentes, estudiantes, administrativos y egresados. Esta cooperación es respaldada por el artículo 63 de la Primera Cumbre de Jefes de Estado y de Gobierno de los países de la Unión Europea, América La- tina, el Caribe (UEALC), donde resalta la voluntad de reforzarla en el ámbito de la educación como reto mayor, insistiendo en la cooperación entre las instituciones de enseñanza superior, respetando su autonomía, especialmente mediante los intercambios universitarios y el desarrollo de la enseñanza a distancia.

Por otra parte, hay que reconocer, con respecto a las relaciones externas, que la participación de docentes y estudiantes en redes académicas y científicas en el contexto internacional ha contribuido a un mejoramiento de los programas. Este hecho, pone de manifiesto la internacionalización como vehículo estratégico de interacción de docentes, estudiantes y egresados con comunidades académicas nacionales e internacionales a través de la ORI y la plataforma institucional como una forma de intercambio y gestión del conocimiento. De acuerdo al "Informe de autoevaluación" y revisado por pares académicos (2016), los convenios suscritos (38 marcos y 12 especializados) y la inversión realizada por la institución para promover los procesos de internacionalización es significativa y valorada para el $100 \%$ de una manera muy favorable entre los integrantes de la comunidad académica. Lo anterior es soportado por los hallazgos de la investigación Internacionalización una visión estratégica de visibilización y cambio institucional en la Universidad de La Guajira (2017) (Fig. 1 y 2).

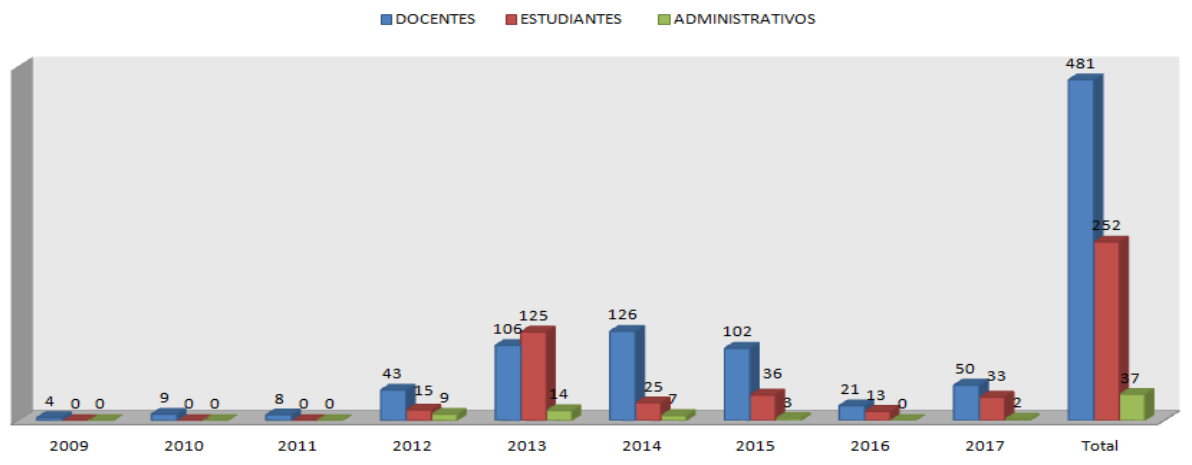

Fig. 1. Movilidad internacional saliente de estamentos UNIGUAJIRA. Fuente: Consuegra y De Luque (2017) 


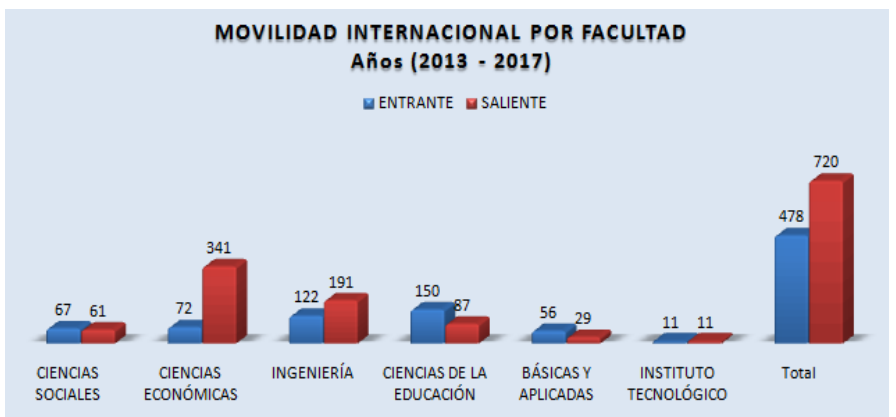

Fig. 2. Comportamiento de la movilidad internacional por facultad: años 2013 - 2017 . Fuente: Consuegra y De Luque (2017)

Los hallazgos del trabajo de campo muestran que para el $54 \%$ de personas participantes del estudio (docentes, estudiantes y administrativos) consideran que la razón más importante que justifica la internacionalización es la internacionalización del currículo y el mejoramiento de la calidad académica, seguida del fortalecimiento de la investigación y la capacidad de producción del conocimiento, y preparar a los estudiantes para un mundo globalizado (37); aspectos estos que contribuyen a nutrir el proceso de acreditación institucional y aseguramiento de la calidad de la educación superior, siendo concordante con el acuerdo de Bolonia y lo expuesto por Atria (2006). En ese orden de ideas, en la medida que se viene posicionado la internacionalización en la misión de la Universidad de La Guajira, se acrecienta su pertinencia en el estamento académico.

Con el fortalecimiento de los vínculos de cooperación y alianzas internacionales entre las comunidades académicas como contribución a la investigación y reproducción del conocimiento, la Universidad de la Guajira abre camino para su visibilidad nacional e internacional de sus programas con miras a consolidar la acreditación, ser competitiva y lograr una articulación positiva en el contexto internacional. Todo esto supone una serie de arreglos y ajustes en el ámbito institucional. De otro lado, para el $100 \%$ de los encuestados de los distintos estamentos, el papel de la ORI en el pro- ceso de acreditación institucional es alto, y coinciden en que sus acciones aportan a la validación del factor de visibilidad nacional e internacional. Esto contrasta con la integración de la dimensión internacional/intercultural en la enseñanza, la investigación y el servicio de la institución (Knight, 2001; Gacel, 2000; De Wit, Jaramillo, Gacel-Avila, y Knight, 2008). La Fig. 2 muestra que hay un predominio de la movilidad saliente.

Del total de la muestra que respondieron la encuesta, un $50 \%$ considera que la mejor estrategia de internacionalización, como aporte a la acreditación, consiste en una mayor financiación para la movilidad saliente, seguida de la suscripción de convenio, con un $31 \%$. Al considerar los avances en la internacionalización, se observa que el establecimiento de las alianzas de cooperación y reconocimiento mutuo con otras instituciones como práctica gerencial ha permitido aumentar su capacidad de gestión internacional. Gracias a ello, es posible avanzar en la transformación curricular logrando una mayor flexibilidad, integración y armonización cultural al igual que la adecuada movilidad trasnacional, teniendo en cuenta la realidad multilingüe, pluricultural y diversa de la Guajira y de toda la región Caribe como característica de la internacionalización. Estas apreciaciones coinciden con los señalamientos de Leask, citado por Jones y Brown (2014); ANUIS (2006); 
Gacel (2000); De Wit, Jaramillo, GacelAvila y Knight (2008) quienes valoran la importancia del reconocimiento cultural en entornos diversos e internacionales. Es así como desde el programa de Licenciatura en Etnoeducación se promueven los diálogos interculturales y el bilingüismo ancestral: el wayuunaiki como lengua importante en el departamento. A su vez, por intermedio de la Escuela de Idiomas, se incentiva, inicialmente, una importante formación en el idioma inglés en docentes y estudiantes, que les permite desempeñarse como profesionales de carácter internacional.

En ese orden de ideas, y recogiendo los intereses, expectativas y prioridades definidas por la comunidad universitaria, las estrategias y acciones que la Universidad de La Guajira ha venido ejecutando se encuentran plasmadas en su Política de Internacionalización (Acuerdo 014 de 2016), siendo lideradas desde la alta dirección y las cuales se registran a continuación:

1. Internacionalización para la calidad académica y la docencia: la docencia, como una de las tres funciones sustantivas de la Universidad de la cual depende en gran medida la labor de enseñanza-aprendizaje, está a cargo de los docentes y se materializa a través de los currículos, así que es la primera que debe replantearse en un proceso de internacionalización con el propósito de garantizar la formación de individuos con base en valores mundiales que estén en capacidad de resolver problemas locales y globales. En este sentido se trabaja en los siguientes ejes:

a. Internacionalización del currículo y de los planes de estudio

b. Formación docente

c. Internacionalización en casa

d. Uso de las tecnologías de la información y la comunicación

e. Gestión de la movilidad

f. Reconocimiento y homologación
2. Herencia, multiculturalidad y multilingüismo: reconociendo su riqueza y legado culturalmente diverso, así como la integración fronteriza con Venezuela, la Universidad de La Guajira se encuentra inmersa en un ambiente multicultural e internacional que busca la inclusión sociocultural de los habitantes de la región. Por esta razón, es necesario que en cualquier política, y más en la de internacionalización, se establezcan estrategias encaminadas a resguardar su identidad, preservando en mayor medida las lenguas nativas y conservando las tradiciones que convergen allí.

a. Enseñanza de lenguas nativas y extranjeras

b. Apropiación y promoción multicultural

3. Visibilidad científica: la investigación como otra de las funciones sustantivas de la Universidad debe contribuir a la producción científica y al desarrollo de conocimiento, aportando soluciones a problemas locales, nacionales y globales. La internacionalización de ésta es indispensable para potenciar la colaboración científica entre investigadores con el fin de impactar realmente en su entorno y visibilizar sus productos a mayor escala. A continuación, se listan las estrategias que se esperan implementar:

a. Gestión internacional de la investigación

b. Fomento a la colaboración científica internacional

c. Promoción a la producción y divulgación científica

4. Gestión y procesos institucionales: la gestión administrativa es esencial para la implementación de cualquier iniciativa académica en una IES, y el proceso de internacionalización no es la excepción, así que este debe contar con el soporte organizacional que conlleva recursos humanos y financieros para facilitar la implementación de estrategias. En este sentido, se espera implementar las siguientes actividades: 
a. Gestión de convenios y redes académicas y científicas

b. Fortalecimiento de procesos de internacionalización

5. Posicionamiento y relación con el entorno: también como una de las tres funciones sustantivas de la Universidad, la extensión o proyección social, se concibe como el relacionamiento con el entorno extendiendo sus acciones académicas y de investigación a la comunidad que la rodea. Así mismo, su internacionalización tiene varios enfoques: la exportación de servicios educativos, la promoción y mercadeo internacional de la institución y la cooperación de proyectos para el desarrollo. Teniendo en cuenta lo anterior, la Universidad de La Guajira tiene un enfoque claro en los siguientes ejes:

\section{a. Proyección internacional \\ b. Promoción e imagen institucional}

Frente a esas consideraciones y consciente de las debilidades, son muchas las expectativas y retos al momento de gerenciar la internacionalización, siendo uno de ellos alcanzar una calificación global óptima en la acreditación institucional. Para ello, es fundamental aumentar el pie de fuerza para apoyar la ejecución del plan curricular con alto reconocimiento e incrementar el número de acuerdos y convenios para el establecimiento de enlaces y contactos con otras instituciones de educación superior a nivel nacional e internacional, e integración a redes que permitan el desarrollo de actividades académicas, investigativas y culturales, así como establecer mecanismos de seguimiento. Conviene subrayar la importancia de revisar sus funciones, así como la dimensión conceptual y los paradigmas que se tejen en torno al aseguramiento de la calidad educativa, internacionalización y acreditación de modo que se puedan redescubrir sus valores, siendo armonizados con sus fines sociales y las estrategias de transformación institucional, por lo que se asume que los planes de mejora, el compromiso y el trabajo cooperativo interno y con otras comunidades académicas de educación superior nacionales e internacionales y demás organizaciones para el desarrollo son el camino para lograr el reconocimiento de la calidad y la alta calidad.

\section{Conclusiones}

Como resultado de la discusión anterior se infiere la relación estrecha entre la internacionalización y la acreditación. En vista que esta última demanda del cumplimiento de factores como la visibilidad nacional e internacional, los procesos de cooperación, colaboración universitaria e integración cultural son necesarios para el desarrollo de los programas de movilidad, de investigación o docencia, requisitos prioritarios para concretar la calidad y, como tal, necesita apoyarse en la internacionalización. En razón a ello, el documento marco Reflexiones para la política de internacionalización de educación superior en Colombia (Salmi y otros, 2014) brinda, como procesos claves que deben liderarse desde el nivel gerencial, una serie de transformaciones relacionadas con la innovación curricular, la movilidad académica y la investigación internacional, los cuales son sujeto de análisis al interior de los resultados.

$\mathrm{Al}$ asumir la cooperación e integración cultural como prácticas gerenciales de internacionalización, se propicia mayor credibilidad y una actitud más positiva frente a lo internacional, asimismo, permite brindar posibilidades de mayor enriquecimiento académico y de apertura a nuevas experiencias internacionales a la comunidad universitaria. En esa dirección, conviene fortalecer la gestión de la calidad promoviendo el establecimiento y apropiación de una política de internacionalización en las esferas políticas y organismos gubernamentales como desafío para darle institucionalidad y, así, favorecer el desarrollo y reconocimiento de la institución en el escenario internacional. 
La Universidad de La Guajira deberá transformarse para ofertar y operar el comercio de servicios educativos con calidad, bien sea bajo la modalidad transfronteriza, consumo de servicios en el extranjero, la prestación de servicios en un país miembro o la prestación de servicios por profesionales extranjeros. Lo anterior está amparado por la Conferencia Regional de Educación Superior de América Latina y el Caribe de 1996, donde se debatió sobre las Estrategias para la Transformación de la Educación Superior en América Latina y el Caribe, tocando como ejes importantes la pertinencia, la calidad, el financiamiento y la gestión de la educación superior, así como la reformulación de la cooperación internacional (Vessuri, 2008; Murga, y Quicios 2006). Como se recuerda, "la calidad educativa tiene como fines establecer mecanismos para asegurar la pertinencia y pertenencia de los procesos de docencia, investigación y extensión (Tobón, Rial, Carretero y García, 2006; ANUIES, 2006).

\section{Referencias}

ANUIS. (2006). Consolidación y avances de la educación superior en México. Elementos de diagnóstico y propuestas. México, D.F.: ANUIS.

Arias, S. y Molina, E. (2008). La cooperación universitaria al desarrollo un desafio permanente. Tabanque, Revista Pedagógica, 20(1), 11-26.

Atria, R. (2006). Modelos de gestión en la educación superior en Chile. El caso de las universidades del Consejo de Rectores. Revista Calidad en Educación Consejo Superior de Educación.

Atria, R. (2012). Tendencias de la educación superior: el contexto del aseguramiento de la calidad. Santiago de Chile: RIL Editores.
Ávila, C. y Aguinaga, C. (2013). El papel de la universidad en el desarrollo de competencias y la acreditación. En V. M. Rosario, A. Didiksson y E. y. Marúm, La acreditación de la Educación superior en Iberoamérica. La gestión de la calidad de los programas educativos, tensiones, desencuentros, conflictos y resultados (Vol. 2). México, D.F.: Palibros.

Bellei, C. (2013). Situación Educativa de América Latina y el Caribe: Hacia la educación de calidad para todos al 2015. Santiago: Ediciones del Imbunche; UNESCO. Recuperado de http://www. unesco.org/new/fileadmin/MULTIMEDIA/FIELD/Santiago/images/SITIEDespanol.pdf

Consejo Nacional de Acreditación-CNA. (2006). Lineamientos para la acreditación institucional. Sistema Nacional de Acreditación. Bogotá, D.C.: Ministerio de Educación Nacional. Recuperado el 16 de julio de 2015, de http://www.cna.gov. co/1741/article-186371.html

Consuegra, M. y De Luque, O. (2017). Oficina de Relaciones Internacionales. La Guajira: Universidad de La Guajira.

CRES (1996). I Conferencia Regional sobre Políticas y Estrategias para la Transformación de la Educación Superior en América Latina y el Caribe. Centro Regional para la Educación Superior en América Latina y el Caribe-IESALC. La Habana, Cuba.

De la Orden, A. (1988). La calidad de la educación. La calidad de los centros educativos, 40(1). 149-161.

De Wit, H., Jaramillo, I., Gacel -Avila, J. y Knight, J. (2008). Educación Superior en América Latina: La dimensión internacional. Washington D.C.: Banco Mundial - Mayol Ediciones.

Gacel, J. (2000). La dimensión internacional de las universidades mexicanas. $R e$ vista de la Educación Superior $y$ Sociedad, 11(1 y 2), 121-142. 
Gacel-Ávila, J. (2012). La educación terciaria en América Latina. El concepto de internacionalización comprehensiva. En H. Grimaldo Dura y F. López Segrera, La internacionalización de la educación superior a nivel mundial y regional (123143). Bogotá, D.C.: Planeta.

Jones, E. y Brown, S. (2014). Internacionalización de la Educación Superior: Perspectivas institucionales, organizativas y éticas. Madrid: Narcea Ediciones.

Knight, J. (2001). Internacionalización de la Educación Superior. En, ANUIES, Calidad e internacionalización de la educación superior (19-35). México, D.F. Recuperado de http://www.ugto.mx/internacional/images/pdf/4a.pdf

Medina, L. y Guzmán, L. (Comp.) (2011). Innovación curricular en instituciones de educación superior. Pautas y procesos para su diseño y gestión. México, D.F.: ANUIES. Recuperado de http://publicaciones.anuies.mx/pdfs/libros/Libro79. pdf

Murga, M. y Quicios, M. (2006). La educación en el siglo XXI. Bordón. Revista de Pedagogía 58(1). 117-119.

República de Colombia. Congreso de la República. (Diciembre 28 de 1992). Por la cual se organiza el servicio público de la Educación Superior. [Ley 30]. Diario Oficial 40.700. Recuperado de https:// www.mineducacion.gov.co/1621/articles-86437_Archivo_pdf.pdf

Rosario, V. M., Marúm, E., Vargas, R., Arroyo, J. y González, V. (2006). Acreditación y certificación de la educación superior: Experiencias, realidades y retos para las IES. Jalisco: Universidad de Guadalajara.

Rosario, V. M., Didiksson, A. y Marúm, A. (2013). La acreditación de la Educación superior en Iberoamérica. La gestión de la calidad de los programas educativos, tensiones, desencuentros, conflictos y resultados (Vol. 2). México, D.F.: Palibros.
Salmi, J., Martínez, P., Nupia, C., LucioArias, D., Lucio, J., Langebaek, C., Téllez, F., Anzola-Pardo, G., Arango, A., Cañón, J., Cruz, V., Toro, S., Vélez, J., De Wit, H., Prieto, L., Valderrama, C., Allain-Muñoz, S. y Aponte, C. (2014). Reflexiones para la política de internacionalización de educación superior en Colombia. (C. Nupia, Ed.) Bogotá D.C.: Ministerio de Educación Nacional - Banco de la República. Recuperado de https:// www.cna.gov.co/1741/articles-186502_ Reflexiones2014.pdf

Tobón, S., Rial, A., Carretero, Á. y García, J. (2006). Competencias, calidad y educación superior. Bogotá, D.C.: Cooperativa Editorial Magisterio.

Universidad Militar Nueva Granada. (2011). Internacionalización. Una Universidad para el mundo. Bogotá, D.C.: UMNG. Recuperado de https://repository.unimilitar.edu.co/bitstream/10654/10616/1/ UniversidadMilitarNuevaGranada.pdf

Vessuri, H. (2008). El conocimiento se enseña, se reproduce y se produce en la universidad. Conferencia Regional para la Educación Superior. Cartagena de Indias, Colombia: UNESCO - Ministerio de Educación Nacional. Recuperado de https://docplayer.es/79226817-El-conocimiento-se-ensena-se-reproduce-y-se-produce-en-la-universidad-vessuri.html

Astrid Terán Molina, PhD en CienciasGerenciales, Docente e investigadora, Universidad de La Guajira

Melissa Paola Consuegra Terán, Msc en Educación, Docente e investigadora, Universidad de La Guajira

Olisney De Luque Montaño, Msc en Cooperación Internacional y Gestión de Proyectos, Docente investigadora, Directora de la Oficina de Relaciones Internacionales, Universidad de La Guajira. 